\title{
Ovarian Mass
}

National Cancer Institute

\section{Source}

National Cancer Institute. Ovarian Mass. NCI Thesaurus. Code C3882.

An abnormal growth in the ovary. 